\title{
Atorvastatin protects endothelium by decreasing asymmetric dimethylarginine in dyslipidemia rats
}

\author{
Dongdan Zheng ${ }^{1}$, Qing Liang ${ }^{2}$, FanFang Zeng ${ }^{3}$, Zhuocheng Mai ${ }^{4}$, Anping Cai ${ }^{1}$, Ruofeng Qiu', Rulin Xu', \\ Dongjuan $\mathrm{Li}^{i^{*}}$ and Weiyi Mai ${ }^{*}$
}

\begin{abstract}
Background: Asymmetric Dimethylarginine (ADMA) is an inhibitor of endogenous nitric oxide synthase, which is the key synthase for nitric oxide (NO) production. Whether statins could protect endothelium by reducing ADMA concentration is unclear, and whether this effect is associated with the dose of statins usage is also needed further studied.

Methods: Dyslipidemia rat model was produced by giving high-fat and high-cholesterol diet for 8 weeks. Thereafter, low-dose (5 mg/kg body weight/day) and high-dose (20 mg/kg body weight/day) atorvastatin were orally prescribed for 4 weeks. Parameters of interest including lipid profiles, inflammatory and oxidative markers, NO production and plasma levels of ADMA and ADMA concentration of myocardium were evaluated. Liver enzymes and creatinine kinase (CK) were also detected for safety concern.

Results: At baseline, all parameters were comparable between the sham and the dyslipidemia groups. At 8 weeks of dyslipidemia establishment, as compared to the sham group, body weight and lipid profiles were significantly elevated, and plasma levels of C-reactive protein (CRP), malondialdehyde (MDA) and ADMA were concomitantly increased in accompanying with $\mathrm{NO}$ reduction in the dyslipidemia groups. With 4 weeks of atorvastatin therapy, as compared to the control group, lipid disorders and NO production were improved, and plasma levels of CRP, MDA and ADMA were significantly decreased in the high-dose atorvastatin group. ADMA concentration of cardiac tissues was also significantly reduced in the high-dose atorvastatin group. Notably, there was a trend to similar effects which did not reach statistical significance in the low-dose atorvastatin group when compared to the control group. Liver enzyme and CK were comparable after 4 weeks of atorvastatin therapy between groups.
\end{abstract}

Conclusion: In rats with dyslipidemia, atorvastatin therapy could reduce plasma level of ADMA and ADMA concentration in cardiac tissues, and these effects are associated with the dose of atorvastatin therapy.

Keywords: Dyslipidemia, Endothelium function, Statins

\section{Introduction}

Atherosclerotic cardiovascular disease (ASCVD) mainly including coronary artery disease and ischemic stroke is still the leading cause of morbidity and mortality worldwide despite great improvement has been achieved in the past decade [1]. As is well known that endothelial dysfunction is implicated in the development and progression of atherosclerosis and ASCVD [2,3], and previous many studies have shown that increased nitric oxide

\footnotetext{
*Correspondence: Idj168168@yeah.net; wymai@hotmail.com

'Department of Cardiology, The First Affiliated Hospital of Sun Yat-sen

University, 58 Zhongshan Road 2, Guangzhou 510080, China

Full list of author information is available at the end of the article
}

(NO) production by statins therapy contributed to better outcomes in experimental and clinical studies [4-6].

Asymmetric dimethylarginine (ADMA), a specific inhibitor of endogenous nitric oxide synthase (eNOS), is capable of decreasing $\mathrm{NO}$ production and may also accelerate atherosclerosis progression as suggested by previous studies [7-9]. For example, Sahinarslan A and colleagues reported that in subjects with stable angina, plasma ADMA concentration was positively correlated with coronary atherosclerotic score [10]. Moreover, increased plasma ADMA concentration might be a good indicator for predicting the severity of coronary artery 
disease [10]. Data from basic research revealed that in the setting of dyslipidemia, ADMA concentration was promoted which consequently leaded to endothelial dysfunction [11]. Outcome from subsequent clinical observational studies further supported the notion that ADMA might be a novel therapeutic target for preventing and treating atherosclerosis and ASCVD [7-9].

Knowingly, dyslipidemia is the major risk factor for atherosclerosis and ASCVD worldwide [12], and statins improved outcomes of ASCVD largely through modifying dyslipidemia as well as improving endothelial function $[13,14]$. However, in the early stage of dyslipidemia, whether statins could protect endothelium by reducing ADMA concentration is unclear yet, and whether this effect is associated with the dose of statins usage is also needed further investigation. Taken together, in order to address the above questions, dyslipidemia animal models were produced and then were treated with different doses of atorvastatin. We considered that the results from our present study could provide evidence regarding the potential therapeutic target of ADMA as a function of prevention and treatment of ASCVD in the future.

\section{Methods}

\section{Animal model production}

Sixty healthy male Sprague-Dawley rats weighing 200$220 \mathrm{~g}$ were obtained from the Experimental Animal Center of Sun Yat-sen University, Guangzhou, China. The study was approved by the Ethic Committee of Sun Yat-sen University. All animals received humane care in compliance with the Guide for the Care and Use of Laboratory Animals of the Institute of Laboratory Animal Resources, National Research Council.

After 1 week of accommodation, dyslipidemia animal model was produced in accordance to the previous described protocol [14]. Briefly, 15 rats were randomly selected and were used as the sham group, in which rats were given diet as usual, and the other 45 rats, evenly and randomly divided into 3 groups $(\mathrm{n}=15)$, were used to produce dyslipidemia model as given with high-fat and high-cholesterol diet consisting of cholesterol (4.0\%), cholic acid (0.4\%), propylthiouracilum $(0.3 \%)$ and lard (10.0\%) for totally 8 weeks.

\section{Protocol of medical treatment}

At 8 weeks of dyslipidemia model production, 5 rats from each group were randomly selected and sacrificed for evaluating the parameters of interest. The rest rats were given different medical treatments for totally 4 weeks. Briefly, rats with dyslipidemia were randomly assigned into the control group (given $3 \mathrm{ml}$ of saline daily orally, $\mathrm{n}=10$ ), the low-dose atorvastatin group $(5 \mathrm{mg} / \mathrm{kg}$ body weight/day of atorvastatin, prescribed orally, $\mathrm{n}=10$ ) and the high-dose atorvastatin group (20 $\mathrm{mg} / \mathrm{kg}$ body weight/day of atorvastatin prescribed orally, $\mathrm{n}=10)$. Rats in the sham group $(\mathrm{n}=10)$ were orally given $3 \mathrm{ml}$ of saline daily.

\section{Parameters of interest assessment}

At baseline, 8 weeks after dyslipidemia model production, and 4 weeks after medical treatment, fasting venous blood in each group were drawn for parameters of interest assessment. Plasma levels of triglyceride (TG), total cholesterol (TC), low density lipoprotein-cholesterol (LDL-C), high density lipoprotein-cholesterol (HDL-C), and fasting blood glucose (FBG) were detected by Automatic Biochemistry Analyzer (Beckman coulter UniCel DxC 800 Synchron). Plasma levels of ADMA (enzymelinking immune-absorbent assay kit, Wuhan Huamei Bioengineering Company, CSB-E13039r), malondialdehyde (MDA assay Kit, Nanjin Jianchen Bioengineering Institute, A003-2), C-reactive protein (CRP assay Kit, Nanjin Jianchen Bioengineering Institute, H151) and NO production (Total Nitric Oxide Kit, Beyotime, Haimen, China, S0023) were evaluated too. Briefly, the plasma level of ADMA was detected by enzyme-linking immune-absorbent assay in accordance to the manufacture's instruction, and the detected wavelength is $450 \mathrm{~nm}$. The intra-assay and inter-assay of coefficient variation were $9 \%$ and $11 \%$ respectively. At 8 weeks of dyslipidemia model production (5 rats from each group were randomly selected) and 4 weeks of medical treatment (the rest 10 rats in each group), after venous blood was drawn, the hearts of rats in each group were harvested for the evaluation of ADMA concentration in cardiac tissues (enzyme-linking immune-absorbent assay kit, Wuhan Huamei Bioengineering Company, CSB-E13039r). Cardiac tissues were homogenized and lysed with $500 \mu \mathrm{L}$ of lysis buffer $(50 \mathrm{mM}$ Tris-HCl, $\mathrm{pH}$ 7.5; $5 \mathrm{mM}$ EDTA; $250 \mathrm{mM} \mathrm{NaCl}$; and $0.1 \%$ Triton X-100) containing $20 \mu \mathrm{L}(10 \mathrm{mg} / \mathrm{ml})$ of protease inhibitors PMSF. Concentration of protein was measured by Bicinchoninic acid (BCA) method. With respect to the safety of statins usage, liver enzymes (alanine aminotransferase, ALT and aspartate aminotransferase, AST) and creatinine kinase (CK) were also detected after 4 weeks of atorvastatin therapy.

\section{Statistical analyses}

Continuous variable was presented as mean $\pm S D$ and compared by the Student's $t$-test when data was normally distributed, otherwise compared by Wilcoxon rank-sum test. Categorical data was presented as percentage and compared by $\chi^{2}$ test. Statistical analyses were performed by using SPSS software version 18.0 (SPSS, Inc., Chicago, Illinois). A value of $\mathrm{p}<0.05$ was considered significant. 


\section{Results}

Baseline characteristics of rats in each group

In order to evaluate the changes of parameters of interest pre- and post-medical treatment, baseline characteristics of rats in each group were evaluated. As shown in Table 1, plasma levels of lipid profiles and FBG were comparable between groups at baseline. In addition, plasma levels of ADMA, MDA and CRP, and NO production were also detected and all the parameters were comparable between groups.

\section{Changes of parameters of interest pre- and post-medical treatment}

Parameters of interests before and after medical treatment were evaluated and compared between groups. As presented in Table 2, body weight and plasma levels of lipid profiles were all significantly increased in the dyslipidemia groups as compared to the sham group, suggesting that dyslipidemia model was successfully established. Of note, FBG level was also concomitantly increased, indicating that in the early stage of dyslipidemia, glucose homeostasis was already impaired. Moreover, parameters of inflammation, oxidation, and endothelial function were also profoundly deteriorated in the dyslipidemia groups. As shown in Table 2, plasma levels of CRP, MDA and ADMA were significantly increased while NO production was profoundly decreased in the dyslipidemia group when compared to the sham group $(\mathrm{p}<0.05)$. Nonetheless, improvements were observed after 4 weeks of medical treatment. Notably, plasma levels of TG, TC and LDL-C were significantly reduced in the high-dose atorvastatin group as compared to the control group, and FBG level was also slightly reduced. Moreover, plasma levels of CRP, MDA and ADMA were significantly decreased, and NO production was profoundly increased in the high-dose atorvastatin group $(\mathrm{p}<0.05)$. Briefly, improvements were also observed in the low-dose atorvastatin group. However, no statistical difference was observed when compared to the control group. After 4 weeks of atorvastatin therapy, no significant differences of ALT, AST and CK levels between groups were observed, suggesting that a short-term of high-dose atorvastatin was safe.

\section{Changes of ADMA concentration in cardiac tissues pre- and post-medical treatment}

At 8 weeks of model production and at 4 weeks of medical treatment, after venous blood sample were drawn, cardiac tissues were harvested and used for ADMA concentration evaluation. As presented in Table 3, at 8 weeks of dyslipidemia model establishment, ADMA concentrations in cardiac tissues were promoted as compared to the sham group, nevertheless, after 4 weeks of atorvastatin treatment, when compared to the control group, ADMA concentration was significantly reduced in the high-dose atorvastatin group $(\mathrm{p}<0.05)$ but marginally decreased in low-dose atorvastatin group $(\mathrm{p}=0.078)$.

\section{Discussion}

Endothelial dysfunction induced by dyslipidemia is implicated in the development and progression of atherosclerosis. Statins, a potent lipid-modified agent, has potent effects on protecting endothelium through multiple mechanisms. Nonetheless, in the early stage of dyslipidemia, whether statins could reduce ADMA and thereby improve endothelial function has not been fully investigated yet. Moreover, whether this effect is associated with the dose of statins is also uncovered. Our present study showed that in rats changing from healthy to morbidity, high-dose of atorvastatin therapy conferred profound protection on endothelium as reflecting by increased NO production and reduced ADMA concentration in both plasma and cardiac tissues. Notably, endothelium-protection with low-dose of atorvastatin therapy did not achieve statistical significance when compared to the control group, suggesting that in the early stage of dyslipidemia, initiating high-dose of statins therapy might promptly prevent endothelial deterioration.

Table 1 Baseline characteristics of rats

\begin{tabular}{|c|c|c|c|c|}
\hline Variables & Sham $(n=15)$ & Control $(n=15)$ & Low-dose $(n=15)$ & High-dose $(n=15)$ \\
\hline Body weight (g) & $215.51 \pm 2.70$ & $213.24 \pm 3.62$ & $216.45 \pm 2.82$ & $215.26 \pm 3.33$ \\
\hline TG $(\mathrm{mmol} / \mathrm{L})$ & $1.03 \pm 0.06$ & $1.06 \pm 0.08$ & $1.03 \pm 0.05$ & $1.04 \pm 0.07$ \\
\hline TC $(\mathrm{mmol} / \mathrm{L})$ & $3.45 \pm 0.11$ & $3.52 \pm 0.14$ & $3.36 \pm 0.15$ & $3.44 \pm 0.13$ \\
\hline LDL-C (mmol/L) & $2.05 \pm 0.10$ & 2. $08 \pm 0.12$ & $2.06 \pm 0.14$ & $2.09 \pm 0.12$ \\
\hline $\mathrm{HDL}-\mathrm{C}(\mathrm{mmol} / \mathrm{L})$ & $0.93 \pm 0.04$ & $0.91 \pm 0.04$ & $0.90 \pm 0.05$ & $0.93 \pm 0.03$ \\
\hline FBG (mmol/L) & $4.06 \pm 0.05$ & $4.10 \pm 0.08$ & $4.08 \pm 0.05$ & $4.10 \pm 0.08$ \\
\hline $\mathrm{NO}$ (umol/L) & $11.73 \pm 1.05$ & $12.02 \pm 1.14$ & $11.81 \pm 1.09$ & $10.98 \pm 1.04$ \\
\hline CRP (mg/L) & $2.16 \pm 0.32$ & $2.28 \pm 0.33$ & $2.17 \pm 0.22$ & $2.32 \pm 0.35$ \\
\hline MDA (nmol/L) & $0.87 \pm 0.04$ & $0.90 \pm 0.06$ & $0.89 \pm 0.03$ & $0.93 \pm 0.05$ \\
\hline ADMA (ng/mL) & $42.52 \pm 6.40$ & $44.75 \pm 5.33$ & $43.90 \pm 6.05$ & $44.15 \pm 5.20$ \\
\hline
\end{tabular}


Table 2 Changes of parameters of interest pre- and post-medical treatment

\begin{tabular}{|c|c|c|c|c|}
\hline Variables & Sham & Control & Low-dose & High-dose \\
\hline \multicolumn{5}{|c|}{ Pre-medical treatment ( 8 weeks of model production, $n=5$ each group) } \\
\hline Body weight (g) & $254.8 \pm 6.6^{*}$ & $286.6 \pm 7.0$ & $290.3 \pm 8.7$ & $288.5 \pm 10.4$ \\
\hline TG (mmol/L) & $1.15 \pm 0.17^{*}$ & $1.79 \pm 0.33$ & $1.78 \pm 0.32$ & $1.76 \pm 0.43$ \\
\hline $\mathrm{TC}(\mathrm{mmol} / \mathrm{L})$ & $3.96 \pm 0.26^{*}$ & $4.97 \pm 0.34$ & $4.96 \pm 0.37$ & $4.95 \pm 0.45$ \\
\hline LDL-C (mmol/L) & $2.12 \pm 0.24^{*}$ & $2.85 \pm 0.31$ & $2.91 \pm 0.57$ & $2.89 \pm 0.44$ \\
\hline $\mathrm{HDL}-\mathrm{C}(\mathrm{mmol} / \mathrm{L})$ & $1.07 \pm 0.12$ & $1.11 \pm 0.04$ & $1.12 \pm 0.04$ & $1.10 \pm 0.05$ \\
\hline FBG (mmol/L) & $4.12 \pm 0.08^{*}$ & $4.49 \pm 0.11$ & $4.51 \pm 0.12$ & $4.50 \pm 0.08$ \\
\hline $\mathrm{NO}(\mathrm{umol} / \mathrm{L})$ & $12.67 \pm 1.37^{*}$ & $9.65 \pm 1.05$ & $9.26 \pm 1.08$ & $9.44 \pm 1.02$ \\
\hline CRP (mg/L) & $2.15 \pm 0.42^{*}$ & $6.37 \pm 0.55$ & $6.52 \pm 0.73$ & $6.46 \pm 0.52$ \\
\hline MDA (nmol/L) & $0.90 \pm 0.06^{*}$ & $2.46 \pm 0.18$ & $2.57 \pm 0.12$ & $2.52 \pm 0.19$ \\
\hline $\mathrm{ADMA}(\mathrm{ng} / \mathrm{mL})$ & $43.37 \pm 6.65^{*}$ & $60.68 \pm 10.17$ & $62.22 \pm 8.17$ & $62.30 \pm 8.22$ \\
\hline \multicolumn{5}{|c|}{ Post-medical treatment ( 4 weeks of medical treatment, $n=10$ each group) } \\
\hline Body weight (g) & $277.3 \pm 8.2^{*}$ & $301.2 \pm 7.4$ & $299.6 \pm 10.3$ & $300.6 \pm 10.5$ \\
\hline TG (mmol/L) & $1.17 \pm 0.22^{*}$ & $1.83 \pm 0.42$ & $1.67 \pm 0.37$ & $1.50 \pm 0.29^{\#}$ \\
\hline $\mathrm{TC}(\mathrm{mmol} / \mathrm{L})$ & $3.98 \pm 0.33^{*}$ & $5.00 \pm 0.62$ & $4.81 \pm 0.56$ & $4.56 \pm 0.36^{\#}$ \\
\hline LDL-C (mmol/L) & $2.13 \pm 0.23^{*}$ & $2.87 \pm 0.36$ & $2.64 \pm 0.52$ & $2.43 \pm 0.35^{\#}$ \\
\hline $\mathrm{HDL}-\mathrm{C}(\mathrm{mmol} / \mathrm{L})$ & $1.07 \pm 0.14$ & $1.10 \pm 0.05$ & $1.12 \pm 0.03$ & $1.10 \pm 0.03$ \\
\hline FBG (mmol/L) & $4.14 \pm 0.07^{*}$ & $4.56 \pm 0.13$ & $4.46 \pm 0.15$ & $4.41 \pm 0.13$ \\
\hline NO (umol/L) & $12.88 \pm 1.56^{*}$ & $9.03 \pm 0.68$ & $10.07 \pm 0.64$ & $10.85 \pm 0.57^{\#}$ \\
\hline CRP (mg/L) & $2.13 \pm 0.39^{*}$ & $6.43 \pm 0.42$ & $5.87 \pm 0.16$ & $5.35 \pm 0.66^{\#}$ \\
\hline MDA (nmol/L) & $0.93 \pm 0.07^{*}$ & $2.52 \pm 0.15$ & $2.29 \pm 0.09$ & $2.05 \pm 0.07^{\#}$ \\
\hline ADMA (ng/mL) & $44.64 \pm 7.08^{*}$ & $62.33 \pm 10.08$ & $58.75 \pm 8.07$ & $54.46 \pm 9.15^{\#}$ \\
\hline ALT (U/L) & $21.43 \pm 3.24$ & $26.55 \pm 5.21$ & $24.47 \pm 6.08$ & $22.39 \pm 3.76$ \\
\hline AST (U/L) & $24.36 \pm 6.77$ & $22.55 \pm 4.09$ & $21.56 \pm 3.35$ & $23.30 \pm 6.12$ \\
\hline CK (U/L) & $18.24 \pm 2.23$ & $21.30 \pm 2.08$ & $20.07 \pm 3.35$ & $20.35 \pm 2.56$ \\
\hline
\end{tabular}

Denote: ${ }^{*} \mathrm{P}<0.05$ versus other groups, ${ }^{\#} \mathrm{P}<0.05$ versus control group.

Currently, statins has been recommended as the cornerstone therapy for the primary and secondary prevention of ASCVD. Other than potent lipid-lowering effect, statins could exert other pleiotropic effects on cardiovascular systems $[15,16]$. For example, statins could ameliorate inflammation and oxidation in rats after acute myocardial infarction as reported by previous basic research [17]. Statins could enhance NO production by stabilizing the mRNA of eNOS [6,18]. Moreover, statins is capable of promoting endothelial progenitor cells migration and facilitating angiogenesis $[19,20]$. Consistent with previous reports $[19,20]$, results from our present study revealed that atorvastatin therapy, with either low-dose or highdose, could reduce plasma levels of CRP and MDA. NO production was also concomitantly enhanced with atorvastatin treatment. Nonetheless, with respect to the statistical significance, only high-dose of atorvastatin therapy achieved significant improvement on endothelial function as compared to the control group.

Knowingly, ADMA is a specific inhibitor of eNOS and many previous research have revealed that increased ADMA level was associated with adverse cardiovascular outcomes [21-23]. The underlying mechanism might be partially attributed to the adverse effects ADMA played

Table 3 Changes of ADMA concentration in cardiac tissues

\begin{tabular}{|c|c|c|c|c|}
\hline Variables & Sham & Control & Low-dose & High-dose \\
\hline \multicolumn{5}{|c|}{ Pre-medical treatment ( 8 weeks of model production, $n=5$ each group) } \\
\hline ADMA ( $\mu \mathrm{mol} / \mathrm{mg}$ protein) & $35.15 \pm 6.03^{*}$ & $48.53 \pm 8.08$ & $50.44 \pm 10.52$ & $49.67 \pm 7.33$ \\
\hline \multicolumn{5}{|c|}{ Post-medical treatment ( 4 weeks of medical treatment, $n=10$ each group) } \\
\hline ADMA ( $\mu \mathrm{mol} / \mathrm{mg}$ protein) & $36.08 \pm 7.43^{*}$ & $51.51 \pm 8.26$ & $47.26 \pm 8.27$ & $41.50 \pm 7.08^{\#}$ \\
\hline
\end{tabular}

Denote: ${ }^{*} \mathrm{P}<0.05$ versus other groups, ${ }^{*} \mathrm{P}<0.05$ versus control group. 
on endothelium $[24,25]$. As is well known that through protecting endothelium, statins exerts its pleiotropic effects on cardiovascular system. Nevertheless, whether these benefits are associated with ADMA reduction is not fully investigated yet. Results from our present study revealed that atorvastatin therapy could reduce both the plasma level of ADMA as well as the ADMA concentration in cardiac tissues in rats with dyslipidemia after 4 weeks' therapy. These benefits were enhanced with high-dose of atorvastatin regimen, while no statistical difference was observed between the low-dose of regimen and the control group, which we considered might be due to the short-term of atorvastatin therapy in the low-dose group as previous many studies have shown that the efficacy of statins therapy was associated with the duration of its usage $[19,20]$. The underlying mechanism associated with atorvastatin therapy on ADMA reduction was not investigated in our present study. However, to the best of our knowledge, we considered some mechanisms might be used to explain our findings. In the first place, it was possible that through modifying dyslipidemia, atorvastatin decreases plasma ADMA level. Data from our present study could provide evidence supporting this notion. As shown in Tables 1 and 2, when compared to baseline, plasma ADMA level was increased in accompanying with lipid disorders after 8 weeks' high-fat and high-cholesterol diet treatment. In contrast, plasma ADMA level was concomitantly reduced when lipid disorders were improved by both lowdose and high-dose of statins therapy. Although there appeared to be a positive relationship between lipid disorders and ADMA change in our present research, we still could not definitely conclude that dyslipidemia was the unique and definitive cause of ADMA elevation. Secondly, since endothelium-protection by statins therapy is evident, it was therefore possible that atorvastatin could antagonize ADMA by directly up-regulating eNOS expression and promoting NO production. In the future, using eNOS knock-out animal model might be helpful to find out whether ADMA reduction by statins therapy was dependent on eNOS/NO signaling pathway. Thirdly, since statins has pleiotropic effects on different signaling pathway, therefore, it was possible that atorvastatin might regulate ADMA metabolism through promoting the activity of dimethylarginine dimethylaminohydrolase (DDAH) or regulating ADMA synthesis through inhibiting protein arginine N-methyltransferase (PRMT). In the future, it is warranted to investigate whether statins has effects on DDAH or PRMT. Last but not the least, the change of ADMA during the initiation and progression of atherosclerosis might be multi-factorial and atorvastatin might directly reduce ADMA level through modulating the gene expression or mRNA transcription.

\section{Conclusion}

In summary, results from our present study show that in rats with dyslipidemia, atorvastatin therapy could reduce plasma level of ADMA and ADMA concentration in cardiac tissues, and these effects are more prominent by high-dose of atorvastatin therapy, suggesting that highdose of statins therapy may confer rapid protection on endothelium, and future study is warranted to investigate whether this early benefit could ultimately turn into long-term clinical benefit.

\section{Competing interests}

The authors declare that they have no competing interests.

\section{Authors' contributions}

$D Z, Q L, F Z, Z M, A C, R Q$ and $R X$ performed this study, DL performed statistic analyses, and DL and WM designed this study and DZ wrote this article. All authors read and approved the final manuscript.

Authors' information

Dongdan Zheng, Qing Liang and FanFang Zeng are co-first authors.

\section{Acknowledgements}

This work was supported by the grants from the Cardiovascular medication grant of Guangdong Province (2011X25).

\section{Author details}

${ }^{1}$ Department of Cardiology, The First Affiliated Hospital of Sun Yat-sen University, 58 Zhongshan Road 2, Guangzhou 510080, China. ²Department of Emergency Medicine, The First Affiliated Hospital of Guangzhou Medical University, Guangzhou 510120, China. ${ }^{3}$ Department of Cardiology, Shenzhen Sun Yat-sen Cardiovascular Hospital, Shenzhen 518000, China. ${ }^{4}$ Division of Biomedical Engineering, School of Engineering, Sun Yat-sen University, Guangzhou 510060, China.

Received: 20 January 2015 Accepted: 24 April 2015

Published online: 02 May 2015

\section{References}

1. Go AS, Mozaffarian D, Roger VL, Benjamin EJ, Berry JD, Blaha MJ, et al. Heart disease and stroke statistics-2014 update: a report from the American Heart Association. Circulation. 2014;129(3):e28-e292.

2. Messner B, Bernhard D. Smoking and cardiovascular disease: mechanisms of endothelial dysfunction and early atherogenesis. Arterioscler, Thromb, Vasc Biol. 2014;34(3):509-15.

3. Horio E, Kadomatsu T, Miyata K, Arai Y, Hosokawa K, Doi Y, et al. Role of endothelial cell-derived angptl2 in vascular inflammation leading to endothelial dysfunction and atherosclerosis progression. Arterioscler, Thromb, Vasc Biol. 2014;34(4):790-800.

4. Cai A, Qiu R, Li L, Zheng D, Dong Y, Yu D, et al. Atorvastatin treatment of rats with ischemia-reperfusion injury improves adipose-derived mesenchymal stem cell migration and survival via the SDF-1alpha/CXCR-4 axis. PLOS ONE. 2013;8(12):e79100.

5. Zheng C, Azcutia V, Aikawa E, Fiqueiredo JL, Croce K, Sonoki H, et al. Statins suppress apolipoprotein CIII-induced vascular endothelial cell activation and monocyte adhesion. Eur Heart J. 2013;34(8):615-24.

6. Stalker TJ, Lefer AM, Scalia R. A new HMG-CoA reductase inhibitor, rosuvastatin, exerts anti-inflammatory effects on the microvascular endothelium: the role of mevalonic acid. Br J Pharmacol. 2001;133(3):406-12.

7. Juonala M, Viikari JS, Alfthan G, Marniemi J, Kahonen M, Taittonen L, et al. Brachial artery flow-mediated dilation and asymmetrical dimethylarginine in the cardiovascular risk in young Finns study. Circulation. 2007;116(12):1367-73.

8. Taner A, Unlu A, Kayrak M, Tekinalp M, Ayhan SS, Aribas A, et al. The value of serum asymmetric dimethylarginine levels for the determination of masked hypertension in patients with diabetes mellitus. Atherosclerosis. 2013;228(2):432-7.

9. Gore MO, Luneburg N, Schwedhelm E, Ayers CR, Anderssohn M, Khera A, et al. Symmetrical dimethylarginine predicts mortality in the general 
population: observations from the Dallas heart study. Arterioscler, Thromb, Vasc Biol. 2013;33(11):2682-8.

10. Sahinarslan A, Cengel A, Biberoglu G, Hasanoglu A, Turkoglu S, Timurkaynak T. Plasma asymmetric dimethylarginine level and extent of lesion at coronary angiography. Coron Artery Dis. 2006;17(7):605-9.

11. Boger RH, Sydow K, Borlak J, Thum T, Lenzen H, Schubert B, et al. LDL cholesterol upregulates synthesis of asymmetrical dimethylarginine in human endothelial cells: involvement of S-adenosylmethionine-dependent methyltransferases. Circ Res. 2000;87(2):99-105.

12. Stone NJ, Robinson JG, Lichtenstein AH, Bairey Merz CN, Blum CB, Eckel RH, et al. 2013 ACC/AHA guideline on the treatment of blood cholesterol to reduce atherosclerotic cardiovascular risk in adults: a report of the American College of Cardiology/American Heart Association Task Force on Practice Guidelines. Circulation. 2014;129(25 Suppl 2):S1-45.

13. Liuni A, Luca MC, Gori T, Parker JD. Loss of the preconditioning effect of rosuvastatin during sustained therapy: a human in vivo study. Am J Physiol Heart Circ Physiol. 2012;302(1):H153-8.

14. Huang C, Cen C, Wang C, Zhan H, Ding X. Synergistic effects of colchicine combined with atorvastatin in rats with hyperlipidemia. Lipids Health Dis. 2014;13:67.

15. Wolfrum S, Jensen KS, Liao JK. Endothelium-dependent effects of statins. Arterioscler, Thromb, Vasc Biol. 2003;23(5):729-36.

16. Antonopoulos AS, Margaritis M, Lee R, Channon K, Antoniades C. Statins as anti-inflammatory agents in atherogenesis: molecular mechanisms and lessons from the recent clinical trials. Curr Pharm Des. 2012;18(11):1519-30.

17. Cai A, Zheng D, Dong Y, Qiu R, Huang Y, Song Y, et al. Efficacy of atorvastatin combined with adipose-derived mesenchymal stem cell transplantation on cardiac function in rats with acute myocardial infarction. Acta Biochim Biophys Sin (Shanghai). 2011;43(11):857-66.

18. Qiu R, Cai A, Dong Y, Zhou Y, Yu D, Huang Y, et al. SDF-1alpha upregulation by atorvastatin in rats with acute myocardial infarction via nitric oxide production confers anti-inflammatory and anti-apoptotic effects. J Biomed Sci. 2012;19:99.

19. Scalia R, Stalker TJ. Microcirculation as a target for the anti-inflammatory properties of statins. Microcirculation. 2002;9(6):431-42.

20. Akhmedov A, Rozenberg I, Paneni F, Camici GG, Shi Y, Doerries C, et al. Endothelial overexpression of LOX-1 increases plaque formation and promotes atherosclerosis in vivo. Eur Heart J. 2014.

21. Bonetti PO, Lerman LO, Lerman A. Endothelial dysfunction: a marker of atherosclerotic risk. Arterioscler Thromb Vasc Biol. 2003;23(2):168-75.

22. Ercan M, Firtina S, Konukoglu D. Comparison of plasma viscosity as a marker of endothelial dysfunction with nitric oxide and asymmetric dimethylarginine in subjects with dyslipidemia. Clin Hemorheol Microcirc. 2014;57(4):315-23.

23. Antoniades C, Shirodaria C, Leeson P, Antonopoulos A, Warrick N, Van-Assche T, et al. Association of plasma asymmetrical dimethylarginine (ADMA) with elevated vascular superoxide production and endothelial nitric oxide synthase uncoupling: implications for endothelial function in human atherosclerosis. Eur Heart J. 2009:30(9):1142-50.

24. Karbach S, Wenzel P, Waisman A, Munzel T, Daiber A. eNOS uncoupling in cardiovascular diseases-the role of oxidative stress and inflammation. Curr Pharm Des. 2014;20(22):3579-94.

25. Siervo M, Corander M, Stranges S, Bluck L. Post-challenge hyperglycaemia, nitric oxide production and endothelial dysfunction: the putative role of asymmetric dimethylarginine (ADMA). Nutr Metab Cardiovasc Dis. 2011:21(1):1-10

\section{Submit your next manuscript to BioMed Central and take full advantage of:}

- Convenient online submission

- Thorough peer review

- No space constraints or color figure charges

- Immediate publication on acceptance

- Inclusion in PubMed, CAS, Scopus and Google Scholar

- Research which is freely available for redistribution 